\title{
Telecommunications Infrastructure to Support Distributed Resources Connected to Electricity Distribution in a Smart Grid Scenario
}

\author{
Leonardo Henrique de Melo Leite ${ }^{1}$, Wallace do Couto Boaventura ${ }^{1}$, Luciano de Errico ${ }^{1}$ \\ ${ }^{1}$ Graduate Program in Electrical Engineering - Federal University of Minas Gerais - Brazil \\ Av. Antônio Carlos 6627 \\ 31270-901 - Belo Horizonte, MG, Brasil \\ lleite@cpdee.ufmg.br, wventura@cpdee.ufmg.br, 1.errico@cpdee.ufmg.br
}

\begin{abstract}
The new concept of smart energy networks - Smart Grids - demands the use of communications solutions that meet critical operational requirements. These requirements are associated with supervision and control functions involved in all stages of electricity supply, especially in distribution systems. This paper discusses the telecommunications infrastructure applied to photovoltaic distributed resources connected to electricity distribution. The goal is to identify, in a Smart Grid environment, strategies to achieve an interoperable supervision system capable to manage photovoltaic plants, in order to cooperate with overall power system performance. The analysis will be based on voltage control scenario.
\end{abstract}

\section{Key words}

Distributed Resource, Photovoltaic Source, Voltage Control, Smart Grid, Telecommunications.

\section{Introduction}

Albeit localized, electric power industry has experienced continuous changes and technological advances, capable of revolutionizing its form of generation, transmission, distribution and consumption. However, to fully benefit from such development, a coordinated and focused effort is necessary to modernize the entire electrical system. In this context, smart energy grids - Smart Grids - arise as a technological model to support the operations and services offered by electricity companies. It proposes a comprehensive architecture, based on open systems, extensive use of sensors, bidirectional telecommunications networks and distributed computing systems. The adoption of this architecture offers many benefits: a more economical and intelligent way to generate, transmit and distribute energy; minimization of environmental impacts; increase of operational efficiency; optimization of energy generation and storage; failure prediction and self-healing and new services to consumers [1].
This paper proposes the design of a communication infrastructure to allow connection of distributed photovoltaic resources to power distribution grid, in a smart grid environment.

Section 2 presents the key points to be considered in the design of smart energy grids, and provides an overview of the main implementation initiatives of this concept in the world. Section 3 discusses the major functions associated with automation of energy distribution, and the related telecommunications aspects. Section 4 presents the advantages of using an operative telecommunications infrastructure to enable the connection of photovoltaic sources to the distribution system, in a safely and efficiently way, based on voltage control scenario. Finally, section 5 brings the final considerations.

\section{Smart Energy Networks}

The electric power system has been often cited as the largest and most complex machine ever built, consisting of cables, towers, transformers, circuits and other devices, all properly connected to provide power distribution services.

However, in some aspects, the basic design of the current electric power infrastructure is still very similar to the one experienced by Thomas Edison and Tesla, in the mid-19th century. Basically, the grid carries large blocks of electricity generated in large centralized plants, mainly based on fossil fuels or hydropower, using a unidirectional transmission and distribution system. Electricity is provided to consumers and devices that have little or no information about its cost or system overload status. The modernization of this system has become a major priority for many countries around the world [2].

The motivation factors for modernizing the electric power system can be summarized as:

i. increase the availability, reliability and quality of the electrical system service; 
ii. generate, transmit and distribute energy in a more safe and cost effective way;

iii. allow a more efficient control of critical points using extensive sensing;

iv. offer new services to consumers as well as information to assist them in making decisions about the rational use of energy;

v. contribute to the reduction of $\mathrm{CO}_{2}$ emissions and other pollutants by intensive use of renewable sources;

vi. integrate alternative energy sources (photovoltaic, wind, biomass, etc.) to the conventional distribution system (medium and low voltage) and enable self-sufficient microgrids in remote regions.

The availability of new technologies, such as two-way communication networks (wired and wireless), smart sensors, autonomous controllers, new forms of energy generation and advanced computational systems, enable a favorable opportunity for the power system transformation.

According to Gharavi and Ghafurian [3] and Moslehi and Kumar [4], among the new features and services to be offered or demanded by this new infrastructure, stand out:

- Self-healing: resilience and automatic network reconfiguration, in order to redirect energy blocks to supply affected regions;

- Fault Location, Isolation and Restoration: ability to more accurately detect the point of failure, to choose the best alternative for isolation and to establish procedures to restore service to sections not affected by the failure;

- Prediction: ability to prevent and recommend actions to reconfigure the system before new harmful events occur, by use of learning techniques, data stochastic analysis and prediction of possible climate changes that can affect the electrical system;

- Optimization: autonomous optimization of the electricity flow through different routes (circuits), by sensing current state of the system operating parameters in real or near real time;

- Flexibility: ability to connect alternative distributed energy sources and energy storage systems at specific points of the electrical system;

- Widespread Supervision and Control: ability to provide appropriate information in a remotely and bidirectional way between grid devices, control systems and operators, in order to enable an optimal and ubiquitous management system;

- Interactivity with Consumers: ability to interact with consumer, enabling it to participate effectively in the demand control cycle, and fit its power consumption based on its individual payment capacity, coupled with incentives from the energy provider (e.g. differentiated charging per hour of use);
- Security: ability to ensure physical and electronic security (cyber security) of critical assets distributed along the grid, considering the acceptable vulnerabilities in an interoperable and communicating scenario.

These new functions and services offered by the Smart Grid architecture represent a paradigm change in the power system functioning. Such a change is granted by the technological advancement of new energy generation forms, by the increase efficiency of transmission and distribution systems and by the personalized relationship with customers.

However, besides its high cost, the design of this new system will need a coordinated action from all directly involved actors - power utilities, industry, research centers, regulatory and government agencies - in an evolutionary implementation process, which can take decades [3].

Perception and significance of these new features and services vary from country to country, according to their immediate modernization needs, medium and long term vision and the current existing technological level.

For example, the USA motivation is an evident need to modernize and increase the reliability of the electrical system, to prevent blackouts as occurred in 2002, which costs amounted to $\$ 79$ billion [4]. In Japan, the goals are on the formation of a "Smart Community", where a new energy system can be fully integrated into society [5]. Japan interruptions in distribution network last on average $16 \mathrm{~min}$ per consumer per year, as opposed to American system, which average is two hours. European countries are interested in expanding the use of renewable energy and in emissions control, in compliance with environmental agenda 20-20-20 for 2020: $20 \%$ emissions reduction, $20 \%$ renewable generation, $20 \%$ energy saving. In Brazil, two major factors drive the deployment of smart grids: the need for increased operational efficiency by energy providers; and the efforts to reduce the high non-technical losses, which in 2010 resulted in a loss of $\$ 8$ billion, equivalent to $8 \%$ of the energy captive market consumption.

\section{Telecommunications Infrastructure}

Smart energy network will become a great system managed and controlled by manual and computerized processes, with data provided by thousands of sensors connected by a wide variety of communication networks. Over the next 20 years, it is estimated that the percentage growth of the data flowing through grid communication networks will far exceed the growth of electricity flowing in the power grids [6].

Energy service providers, whether power generation, transmission or distribution, already use some type of communication network to support different services: $i$ ) proprietary networks for data communication between automation field devices and supervisory centers, $i$ ) private networks, for the same purpose, but also for communication between enterprise sites and iii) public networks (e.g. fixed telephony networks, mobile and internet) used in non-critical operational processes and as data backbone for enterprise services. 
However, the design of smart energy networks and new advanced functions will require an extensive, faster, more flexible and more reliable communication infrastructure. The current point to point network should be replaced by bidirectional networks, with broadcasting capability.

Figure 2 illustrates a conceptual representation of communication flow in this new scenario, considering transactions between generation, transmission and distribution agents, communication with consumers and contractors, and interactions with the market. The entire energy supply chain becomes interconnected to local, regional and metropolitan communication infrastructures.

Critical supervision and control functions will have access to several devices and sensors distributed in the energy infrastructure, using dedicated or shared data communication networks.

Market agents and outsourced service providers can use the internet for communication with the energy enterprise systems.

The consumer, who previous role was only to consume the electricity service, becomes an active participant agent. New features (e.g. distributed generation, home automation, electric vehicles, remote metering, etc.) require new direct communication interfaces with consumers, enabling them to interact directly with their electricity provider.

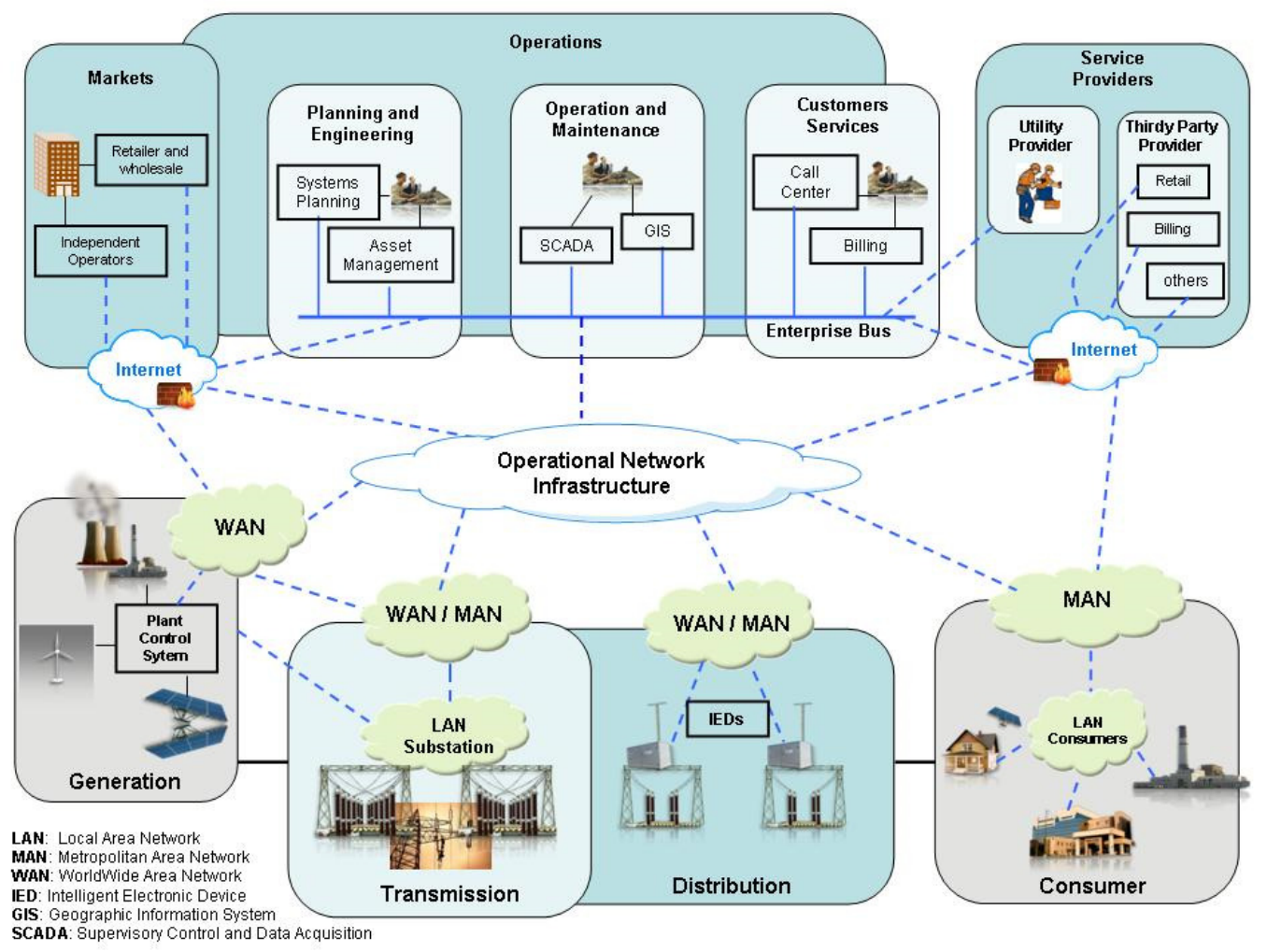

Fig. 2. Proposed New Flow Communication

Source: adapted from - National Institute for Standards and Technology [7]

\section{A. Data Communication Technologies}

In the last decade, there has been a great progress in data communication technologies, each focused on specific applications and different coverage areas.

The choice of the best communication technology applied to the electric power infrastructure will depend on communication requirements (data rate, latency, coverage, availability, quality of service, security) and the geographical area of interest. Different electrical system functions will present different requirements, some more restrictive, others more flexible. For example, functions associated with metering automation process require a relatively low data rate per measuring point, around 100 kbps. On the other hand, those related to supervision and control processes of vital power system parameters (voltage, current, phase, frequency) will require a network with high baud rate (> $1 \mathrm{Mbps}$ ), enabling massive sensing data and real time control actions.

The network response time is also a critical parameter. For example, functions related to the power system protection act with maximum latency of $20 \mathrm{~ms}$, while others are much less restrictive.

Another sensitive requirement is the communication network availability. Some less critical functions require $99 \%$ of availability (interruption tolerance of 3.65 days per year). Other, related to network distribution management and synchronization between elements of this network, will require an availability up to $99.9999 \%$ (interruption tolerance of only 31 seconds per year).

Due to different needs, related to connectivity parameters and different geographical topology, there is no single 
technology solution for the communication network that will supply all demands. The major wireline and wireless presently available technologies should be technically and economically analyzed according to specific scenarios.

\section{B. Proprietary $x$ Public Networks}

The communication network ownership that supports operational functions of electric utilities has been subject of frequent controversy among the designers: invest in proprietary infrastructure or use the services offered by telecommunications companies.

Traditionally, electric power companies have designed, built and operated its own communication infrastructure, to support the critical operational functions sensitive to response time, reliability and security requirements. The commercial communication networks are typically used only for less critical functions and to those used in enterprise services.

However, due to the large area covered by some utilities and the growth trend of sensed points on the grid, there will be an increasingly demand for a capillary and complex communication infrastructure, resulting in a high cost to deploy, maintain and operate it.

For the other hand, the advancement of communication technologies used by telecom companies (public or private), whose performance, security and manageability have shown significant improvements, coupled with falling costs, provide a new alternative for energy companies, even for critical services support. Although, in this scenario, the physical medium is shared by multiple applications and by different enterprises, telecom companies claim to ensure QoS (Quality of Service) parameters, agreed in contract.

A question must be answered: how a shared infrastructure will behave in an emergency, where network is overloaded by several requests? Critical remote supervisory operation may be requested in those exactly moments.

Although several tests must still be performed, the choice of purchase model will depend on assessment of each company, regarding to operational requirements restriction, investment analysis (assets acquisition $\mathrm{x}$ services contracting), regulatory issues and safety.

Pilot projects involving a mix of telecommunications solutions have been conducted to verify the best solutions, both from a technological and commercial point of view. Probably, there will not a unique solution or model.

\section{Cyber Security}

In recent years, the electric power industry has shown a growing concern with the reliability of power systems, which has led to questions related to their safety, highlighted by several instances of cyber attacks in many energy installations worldwide.

In the current scenario, the reliability of these systems is not only threatened by equipment failures, adverse weather conditions, natural disasters and quick increase of consumption of electrical energy, but also by cyber terrorists, whose goal is to interrupt the electricity supply through illegal access to generation, transmission and distribution assets.

Cyber security in the electric power industry represents a great challenge, because systems based on SCADA/EMS (Supervisory Control and Data Acquisition System / Energy Management System) and DCS (Distributed Control Systems) are designed prioritizing efficiency and reliability, instead of security. Many of these systems even lack basic security mechanisms, especially compared with current management enterprise systems [8].

Energy companies should adopt, beyond technologies, a structured security plan that control and restrain risks automatically. Only with security policies is possible to have proactive responses and adaptation to vulnerabilities.

\section{Telecom Infrastructure to Support Photovoltaic Distributed Resource}

The planning of the electric system with the presence of DG (Distributed Generation) requires the definition of several factors, such as: the best technology to be used, the number and the capacity of the units, the best location, the network connection way, etc. The impact of DG in system operating characteristics, such as electric losses, voltage profile, reliability, among other, needs to be appropriately evaluated. The selection of the best places for installation and the size of the DG units in large distribution systems is a complex combinatorial optimization problem [9].

When large photovoltaic generation plants are directly connected to a distribution feeder that serves a range of loads, consideration about the ability of these sources offering ancillary services in a contingency or disturbance situations (e.g. voltage regulation ), should be considered.

\section{A. Voltage Control Issues}

The protections of the electrical system against over current and voltage variations are traditionally designed in a unidirectional power flow, from the substation toward the load. Voltage variation in steady state and due to shortterm events, such as those caused by short circuits, can cause significant changes in the voltage profile along a feeder, which large blocks of load are connected. Techniques and protective devices such as voltage regulators, automatic tap changing transformers and switching capacitor banks are normally used to control the voltage profile in the distribution network. Given this new scenario, where multiple photovoltaic sources are connected to the distribution bus, some studies show the ability of these sources to regulate the voltage at point of interconnection, from advanced control techniques employed by their inverters devices. However, voltage regulation has been taken in a reactive way, locally and individually by each photovoltaic source. 
Voltage variation at a given point caused by the insertion of photovoltaic power generation can be expressed in a simplified form by equation (1).

$$
\Delta V \approx \frac{\Delta P}{V} \cdot R-\frac{\Delta Q}{V} \cdot X
$$

$\Delta V=$ voltage variation at the interconnection point

$V=$ voltage at the interconnection point

$\Delta P=$ active power variation at the interconnection point

$\Delta Q=$ reactive power variation at the interconnection point

$P=$ active power

$Q=$ reactive power

$R=$ resistence at the interconnection point

$X=$ reactance at the interconnection point

In equation (1) setting the voltage variation $\Delta \mathrm{V}$ to zero and solving for $\Delta \mathrm{Q}$, we have:

$$
\Delta Q \approx \Delta P \cdot \frac{R}{X}
$$

Equation (2) shows that injection of reactive power by photovoltaic source can be used to nullify the voltage variation at interconnection point, once known behavior of impedance $\mathrm{R}+\mathrm{j} X$ at this point [10].

Simulations using PSCAD (Power Systems Computer Aided Design) indicate the potential contribution of photovoltaic sources to control the voltage profile during events caused by short circuits [11].

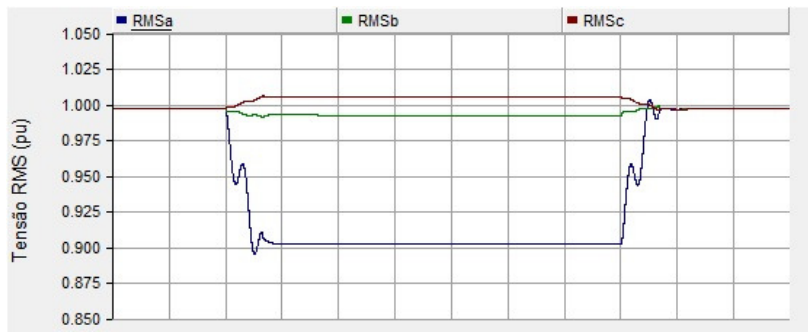

Fig. 3 (a) Without Photovoltaic DG

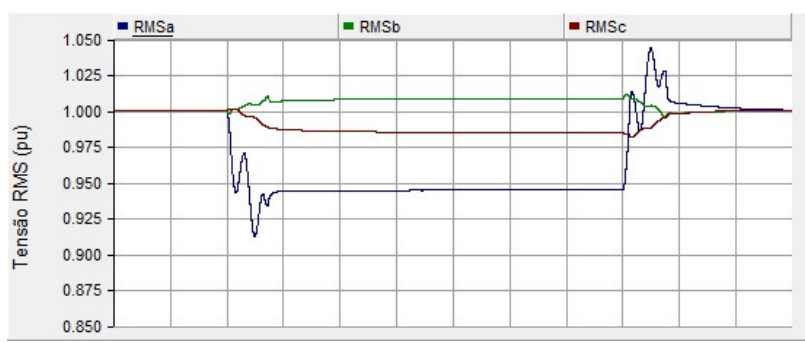

Fig. 3 (b) With Photovoltaic DG

Scenarios of phase-to-ground fault, phase-to-phase and three-phase faults over a $13.8 \mathrm{KV}$ feeder with $20 \%$ penetration of photovoltaic were simulated. The curves plotted in Figure 3 show an example of recovery voltage level after occurrence of phase-to-ground fault, from the injection of reactive power by photovoltaic sources.

\section{B. Telecommunication System to Coordinate FV Sources on Grid Voltage Control Support}

When multiple photovoltaic plants are connected at different points in the distribution system, the challenge is to determine, in real or near real time, the contribution of each of them on voltage profile control along the feeder.

This control should be carried out from the controlled injection of reactive power for each of photovoltaic sources without overly compromising to supply active power to critical loads.

Issues related to the best places for installation, the ability to supply active and reactive power associated to photovoltaic generation predictability in adverse situations must be considered.

Current Distribution Management Systems (DMS) are not designed to manage and operate the photovoltaic generation plants. In some cases, the control is quite limited not allowing the benefits of an integrated and broader control involving distributed generation sources. Therefore, it is necessary that DMS expand their roles by integrating a Distributed Generation Management Systems (DGMS).

The DGMS can manage various photovoltaic sources connected to the grid and make the interface with the DMS to necessary transactions. From these interactions, the DMS can make decisions based on effects caused by DG sources in addition to existing assets in the current distribution network. On the other way, DMS requests DGMS to act on DG sources in order to cooperate for achieving the desired operational levels.

This integration scenario is only possible through an operational telecommunications system that enables transactions among various DG sources, DGMS and DMS, as illustrate in Figure 4.

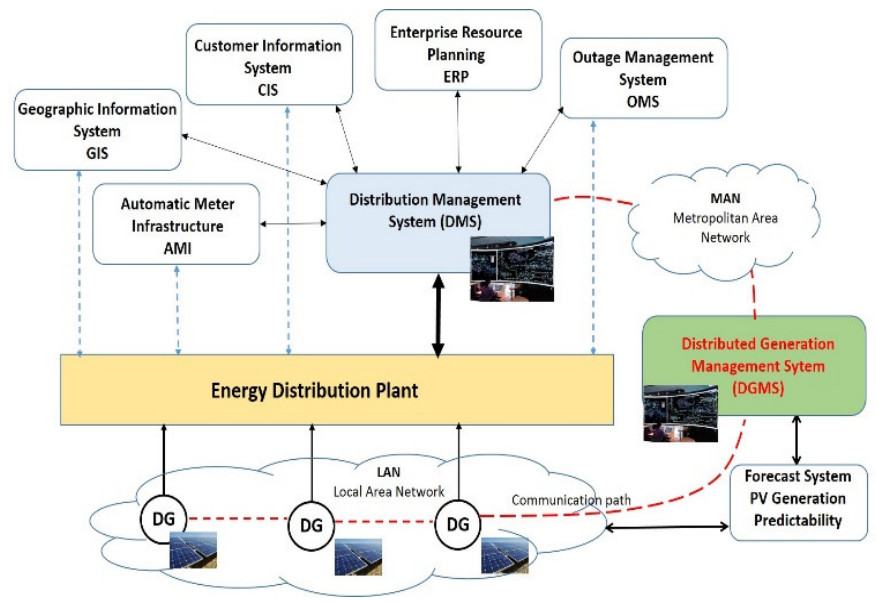

Fig. 4. DMS x DGMS Integration Architecture

The topology of the communication system and the main connectivity requirements such as response time and data communication rate must be properly specified and designed to enable this integration. 


\section{Conclusions}

The deregulation of the energy market, the need to adapt to the "digital society" demands and the changing of electric load profile have forced electricity companies (generation, transmission and distribution) to be more competitive and adaptable. This necessarily involves the integration of its enterprise and operating systems, making them flexible, extensive and interoperable. This huge task has been tackled by implementing the Smart Grids.

The design of intelligent energy networks - Smart Grids requires the use of communication solutions that meet critical operational requirements, associated with supervision and control functions involved in all stages of the electricity supply, especially in distribution systems. As there will be no single communication architecture, which is the solution to all processes, different network topologies, with local or metropolitan coverage, and related technologies must be integrated.

The ability of photovoltaic generation plants offering ancillary services such as voltage regulation should be considered in a Smart Grid scenario.

However, only through an advanced communication infrastructure that enables the integration of various photovoltaic sources and management systems, it will be possible the full realization of these services.

\section{Acknowledgement}

This work has been supported by the Brazilian agency CAPES. The authors would also like to thank FAPEMIG and $\mathrm{CNPq}$ for their financial support to this research.

\section{References}

[1] Gellings, C. W. (2009). The Smart Grid - Enabling Energy Efficiency and Demand Response. Boca Raton, FL, USA. Taylor \& Francis, 2009, 300p.

[2] Arnold, G. W. (2011). Challenges and Opportunities in Smart Grid: A Position Article. Proceedings of the IEEE, Vol.99, No. 6; pp. 922-927.

[3] Gharavi, H. and Ghafurian, R. (2011). Smart Grid: The Electric Energy System of the Future. Proceedings of the IEEE, Vol.99, No. 6; pp. 917-921.

[4] LaCommare, K. H. and Eto, J. H. (2006). Cost of power interruptions to electricity consumers in the United States. LBNL - 58164, 2006.

[5] Nakanishi, H. (2010). Japans's approaches to smart community. Present at the First IEEE Conference on Smart Grid Communication. Oct. 4-6, 2010. Available in: http://www.ieeesmartgridcomm.org/2010/download s/Keynotes/nist.pdf

[6] MIT (2011). The Future of the Electric Grid - An Interdisciplinary MIT Study. Massachusetts Institute of Technology, 2011, 268p.

[7] NIST (2011). National Institute for Standards and Technology, NIST Framework and Roadmap for
Smart Grid Interoperability Standards, Release 1.0. Special Publication, January, 2011.

[8] Leite, L.; Heinisch, A. ; Speyr, B. ; Rabello, M. (2011). Segurança Cibernética para Processos Operativos em Sistemas de Energia Elétrica - VI Congresso de Inovação Tecnológica em Energia Elétrica (VI CITENE, August, 17, 2011, FortalezaCE.

[9] Borges, C. and Falcão, D. (2003). Impact of Distributed Generation Allocation and Sizing on Reliability, Losses and Voltage Profile. IEEE Bologna Tech Conference. June 23th-26-th, Bologna, Italy. 2003.

[10] Walling, R. and Clark, K. (2010). Grid Support Functions Implemented In Utility-Scale PV System. IEEE PES Transmission and Distribution Conference and Exposition, 2010.

[11] Silva, T; Boaventura, W; Leite, L. (2013). Análise da Contribuição de Geração Distribuída Fotovoltaica para o Controle do Perfil de Tensão Frente a Variações de Tensão de Curta Duração. Trabalho de Conclusão de Curso de Engenharia Elétrica. Belo Horizonte, Dezembro, 2013.

\section{Biographies}

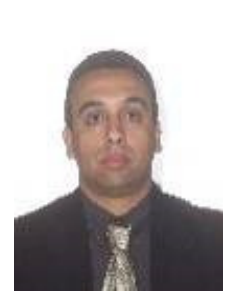

Leonardo Leite is a $\mathrm{PhD}$ student in Electrical Engineering at Center for Research and Development in Electrical Engineering from Federal University of Minas Gerais - UFMG. He graduated in Electrical Engineering (2000) and obtained masters degree in Computer Engineering and Telecommunications (2005) at the same university. He is also senior engineer at FITec Fundação para Inovações Tecnológicas. His current research focuses on energy resources, telecommunications and high performance automation systems.

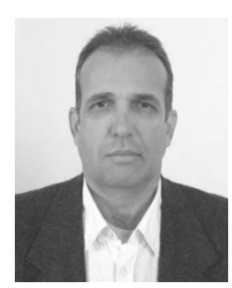

Wallace Couto Boaventura has degree in Electrical Engineering from Federal University of Minas Gerais (1987), Masters in Electrical Engineering from Federal University of Minas Gerais (1990) and Ph.D. in Electrical Engineering from the State University of Campinas (2002). He is currently Associate Professor at the Federal University of Minas Gerais. He works in area of signal processing applications in power systems and electromagnetic compatibility, addressing issues related to Parametric Modeling, Grid Equivalents, Electromagnetic Transients, Power Systems, High Voltage and Lightning.

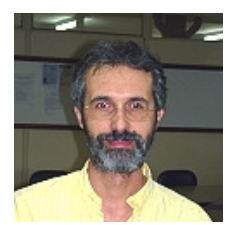

Luciano Errico has degree in Electrical Engineering from UFMG (1983), Masters in Computer Science from Department of Computer Science, UFMG (1991) and PhD in Electronic and Electrical Engineering from the University of Surrey - UK (1996). He is currently Associate Professor in Department of Electronic Engineering, UFMG, where he teaches subjects related to Computer Networks and TCP / IP Network to undergraduate and postgraduate students. He has experience in the area of Computer Engineering, with emphasis in Computer Networks and Distributed Systems, acting on the following topics: Traffic Engineering in IP Networks, Networks Self Manageable (Autonomic), Multimedia Communication over IP (VoIP, IPTV) and Distributed Process. 